\title{
ANÁLISE E COMPARAÇÃO DE ESTRATÉGIAS DE PREVISÃO MULTIPASSOS À FRENTE PARA VAZÃO AFLUENTE EM BACIAS HIDROGRÁFICAS
}

\author{
SAMIRA D. COSTA ${ }^{1}$, EDUARDO M. A. M. MENDES ${ }^{2}$.
}

\author{
1. Laboratório de pesquisa MACSIN, Departamento de Engenharia Eletrônica, Universidade Federal de \\ Minas Gerais \\ av. Antônio Carlos, 6627,31270-901, Belo Horizonte, MG, BRASIL \\ E-mails: samiradcmeufmg.br, mirinhacostalyahoo.com.br
}

\author{
2. Laboratório de pesquisa MACSIN, Departamento de Engenharia Eletrônica, Universidade Federal de \\ Minas Gerais \\ av. Antônio Carlos, 6627,31270-901, Belo Horizonte, MG, BRASIL \\ E-mails: emmendes@cpdee.ufmg.br, emammendes@gmail.com
}

\begin{abstract}
One of the problems in the time series literature is to obtain multistep ahead predictions with good accuracy. In order to achieve this goal, not only the algorithm used but the strategy applied is of great importance. In this work, to improve the performance of the estimation algorithm, five different strategies for MultiStep ahead predictions, Direct, Recursive, DirRec, MIMO and DIRMO are compared and analyzed in the context of a real prediction problem. The piece of real data used here contains precipitation and riverflow measurements of a small hydroelectric power station in the state of Minas Gerais, UHE Rosal Cemig. The results show that the Recursive strategy performed better while the strategy DirRec exhibits the best MAPE index.
\end{abstract}

Keywords_- Prediction, Multi-step, Time-Series

\begin{abstract}
Resumo- A obtenção de uma boa Predição de Multipassos à frente é um dos grandes objetivos em diversas áreas da ciência que lidam com com séries temporais. Para alcançar este objetivo, não só o algoritmo utilizado bem como a estratégia aplicada para a determinação da predição devem ser considerados. Neste trabalho, visando obter o melhor desempenho do algoritmo utilizado no difícil problema de predição de séries reais de vazão, discute-se cinco estratégias de predição multipassos à frente hoje disponíveis na literatura técnica: Direta, Recursiva, DirRec, MIMO e DIRMO. Os dados utilizados, precipitação e vazão, são reais e referem-se a uma bacia hidrográfica de pequeno porte. Os resultados obtidos apontam que a estratégia Recursiva teve melhor desempenho mas a estratégia DirRec obteve o melhor índice MAPE.
\end{abstract}

Palavras-chave—Predição, Multipassos, Séries Temporais

\section{Introdução}

Previsão multipassos à frente de séries temporais é um grande desafio em diversas áreas do conhecimento tais como economia (Chevillon, 2007, Marcellino et al., 2005) e hidrologia (Bhagwat et al., 2012, Pionosi e Raso, 2012, Bao et al.,2012), pois quanto maior o horizonte de previsão, maior é a incerteza (Ben Taieb et al., 2011).

Apesar de autores como Box and Jenkins (2008) apresentarem diversas ferramentas para modelagem e análise de séries temporais, além de métodos para construção, classificação, testes e análises de modelos estocásticos e consequentemente métodos para predição, vê-se, na literatura especializada, crescente necessidade de estudos sobre a melhor estratégia a ser aplicada para predição a longo prazo de séries temporais (Ben Taieb et al. , 2011).

A idéia central é analisar diferentes estratégias de previsão para multipassos à frente, uma vez que não só o algoritmo é importante, mas a escolha correta da estratégia também pode influenciar no desempenho do mesmo. Sorjama e Lendasse (2006) de- monstram como estratégias diferentes são importantes para predição de séries temporais, assim como Ben Taieb et al. ( 2011).

Ben Taieb et al. (2011) afirmam que uma de suas contribuições é apresentar uma revisão, acompanhada por uma comparação teórica das estratégias de previsão existentes para multipassos à frente, com o objetivo de verificar o desempenho de cada uma delas. Eles também fazem uma comparação experimental na qual utilizaram o algoritmo de Lazy Learning (LL) na série temporal 111 da competição internacional de previsão do NN5. Os resultados obtidos apontam que as abordagens utilizando um modelo multi-saídas é melhor do que aquelas que utilizam uma única saída. Foi constatado ainda que técnicas de pré-processamento dos dados, como por exemplo a dessazonalização, podem levar a um melhor desempenho quando se considera a previsão de vários passos à frente.

Ben Taieb et al. (2009) comparam as estratégias Diretas e MIMO (Multi-Input Multi-Ouput), discutindo suas limitações para o problema de predição a longo prazo de séries temporais. Neste mesmo trabalho eles propõem uma nova metodologia que é uma 
espécie intermediária entre a estratégia Direta e MIMO, denominada DIRMO, buscando preservar os melhores aspectos das duas estratégias. Os resultados iniciais demonstraram que esta abordagem é promissora.

O presente artigo propõe aplicar e analisar cinco tipos de estratégias (Direta, Recursiva, MIMO, DirRec, DIRMO) no problema de previsão de séries de vazão real.

Na Seção 2 serão apresentadas as 5 estratégias para predição multipassos à frente. Na Seção 3, uma breve descrição da estratégia de modelagem utilizada neste trabalho e dos dados utilizados na mesma é dada. Nessa mesma seção vários índices de desempenho de modelos para posteriormente comparação são dados. Os resultados e a discussão dos mesmos são apresentados na Seção 4. Finalmente na Seção 5 as conclusões são apresentadas.

\section{Tipos de estratégias para predição de multi- passos a frente}

Nesta seção serão apresentadas as várias estratégias para predição de multipassos à frente.

\subsection{Estratégia Recursiva}

A estratégia Recursiva, também conhecida como Iterada, pode ser considerada como a estratégia mais antiga e intuitiva conforme relatado por Cheng et al. (2006) e Sorjama et al. (2007). Nela um único modelo $(f)$ é treinado para executar predição de um passo à frente. Sorjama e Lendasse (2006) explicam em seu trabalho que o mesmo modelo é utilizado para as próximas predições, sendo que a anterior passa a ser entrada do passo seguinte e assim sucessivamente de forma que a predição de um passo à frente é aplicada muitas e muitas vezes, de forma recursiva. A estratégia Recursiva para três passos à frente pode ser exemplificada pela seguintes equações (1):

A grande desvantagem é que a estratégia Recursiva é sensível à acumulação de erros com o horizonte de previsão (Taieb et al., 2012).

\subsection{Estratégia Direta}

Diferentemente da estratégia Recursiva a estratégia Direta não utiliza recursão no cálculo das predições, impedindo assim a acumulação de erros introduzidos na primeira. A estratégia Direta utiliza diferentes modelos para cada passo de predição, mas sempre tendo como entrada os dados reais medidos (Sorjama e Lendasse, 2006). A estratégia Direta pode ser descrita pelas equações 2 abaixo:

Como descrito em (Sorjama e Lendasse, 2006), nota-se que a cada passo de tempo um modelo é incorporado com entradas e estrutura próprias. Em princípio, acredita-se que o aumento de complexidade, devido ao número de modelos a serem identificados e que depende do número de passos de predição desejado, pode ser compensado pela ausência de erro acumulativo.

\subsection{Estratégia DirRec}

A estratégia DirRec pode ser entendida como a junção da Recursiva e da Direta, combinando os melhores aspectos de ambas. A DirRec utiliza um modelo diferente para cada passo, similar a Direta, e atualiza o conjunto das variáveis de entrada com os valores das predições anteriores, como na Recursiva. Ela pode ser escrita pelas seguintes equações (3):

\subsection{Estratégia MIMO}

A estratégia MIMO, múltiplas entradas e múltiplas saídas, foi introduzida por Bontempi (2008) e Bontempi e Ben Taieb (2011) em seus trabalhos. Diferente das anteriores em que tinham apenas uma única saída.

Na estratégia MIMO apenas um único modelo $(f)$ de múltiplas entradas e múltiplas saídas é identificado. A estratégia surgiu com o intuito de evitar a modelagem com uma única saída na qual se faz necessário saber a dependência com valores ainda não conhecidos. Desse modelo acredita-se que há diminuição ou mesmo eliminação das dependências estocásticas entre valores futuros.

A estratégia MIMO pode ser representada conforme mostrado abaixo (Eq. 4):

onde $H$ é o horizonte de predição e $d$ o atraso máximo do modelo escolhido.

Esta estratégia evita a independência condicional, suposição feita pela estratégia direta, bem como a acumulação de erros como na recursiva (Ben Taieb e Bontempi, 2012). Segundo os autores, ela tem sido aplicada com sucesso em problema de predição de séries reais. A necessidade de preservar as dependências estocásticas usando um único modelo tem sua desvantagem, uma vez que restringe todos os horizontes a serem previstos com uma mesma estrutura de modelo. Essa desvantagem é a principal motivação para o surgimento de uma nova estratégia: DIRMO. 


\subsection{Estratégia DIRMO}

A estratégia DIRMO visa preservar os aspectos mais atraentes das estratégias Direta e MIMO. Tomando uma abordagem intermediária, DIRMO prevê o horizonte $H$ em blocos, onde cada bloco é previsto de forma MIMO. Assim a tarefa de previsão de $H$ passos à frente é decomposta em $n$ previsões de múltiplas saídas $(n=H / s)$ e cada saída de nho $s$ (Sorjama e Lendasse, 2006). Sendo $N$ o número de observações e $p$ o número de modelos.

$[y(t+p * s), \ldots, y(t+(p-1) * s+1)]=f_{p}(y(t), \ldots, y(t-d+1))$

onde $t \in\{d, \ldots, N-H\}, p \in\{1, \ldots n\}$ e $f_{p}: \mathbb{R}^{d} \rightarrow \mathbb{R}^{s}$ se $s>1$.

As $H$ previsões são retornadas pelos $n$ modelos de aprendizagem (Eq. 6):

$[\hat{y}(t+p * s), \ldots, \hat{y}(t+(p-1) * s+1)]=\widehat{f}_{p}(y(N), \ldots, y(N-d+1))$

Conforme explicado pelos autores, quando o valor de $s$ é 1 , a quantidade de previsões $n$ é igual a $H$, correspondendo a estratégia Direta. E quando o valor de $s$ é $H$, o número de previsões $n$ é equivalente a 1 , correspondendo a estratégia MIMO. Entre os dois extremos, tem-se uma configuração intermediária dependendo do valor de $s$.

\section{Modelagem de Séries Temporais}

\subsection{Descrição dos dados utilizados na Modelagem}

O dados utilizados neste trabalho são de uma bacia hidrográfica de pequeno porte, UHE Rosal no estado de Minas Gerais, e representam valores diários de precipitação e de vazão. Por ser de pequeno porte e consequentemente de dinâmica rápida quando comparada a bacias como São Simão e outras, a amostragem utilizada pode comprometer a predição. Foram utilizados como dados de entrada os valores de precipitação e de saída os valores de vazão. Os mesmos referem-se ao período do dia 26 de maio de 1998 a 31 de março de 2012.

As Figuras 1 e 2 mostram as séries temporais de precipitação real e vazão afluente para a UHE Rosal no estado de Minas Gerais.

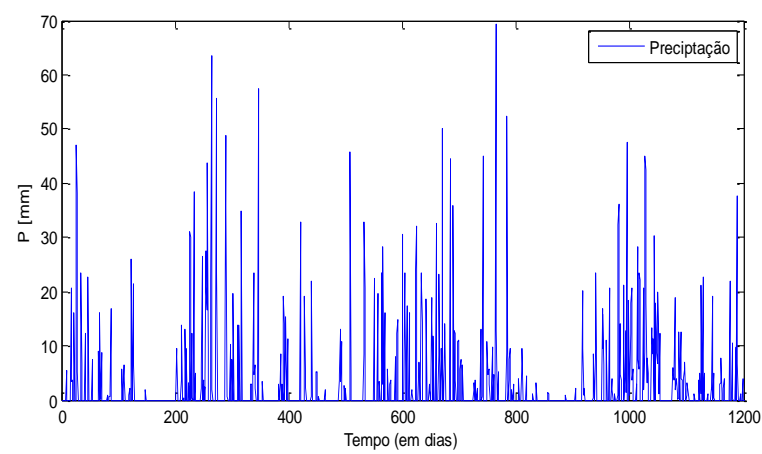

Figura 1: Série temporal de precipitação P (dada em mm).

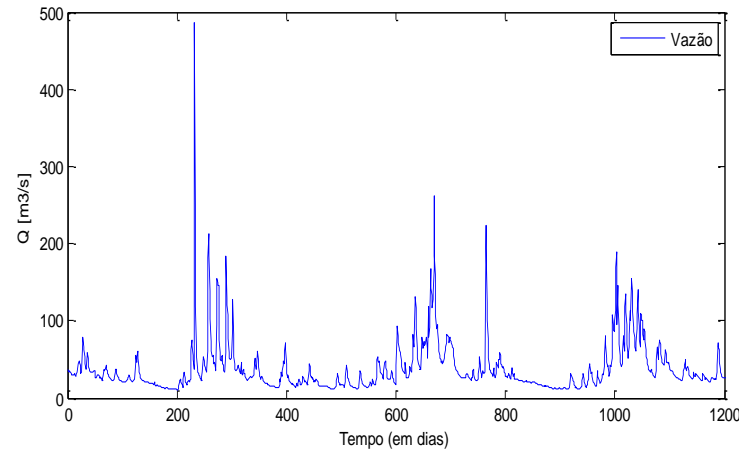

Figura 2: Série temporal de vazão Q (dada em m3/s).

\subsection{Modelagem ARMAX}

O objetivo deste trabalho não é analisar a melhor estratégia aplicada na etapa de modelagem da série temporal e sim qual é a melhor estratégia para se fazer predições multipassos à frente. Diante disso, optou-se pela modelagem ARMAX (AutoRegressive Moving Average with eXogenous inputs model) por esta ser amplamente utilizada na literatura especializada.

Utilizando (Aguirre, 2007) a equação do modelo ARMAX pode ser escrita da seguinte forma (Equações 7 e 8):

$$
\begin{gathered}
A(q) y(k)=B(q) u(k)+C(q) v(k) \\
\text { ou } \begin{array}{c}
y(k)=\frac{B(q)}{A(q)} u(k)+\frac{C(q)}{A(q)} v(k) \\
y(k)=H(q) u(k)+e(k)
\end{array}
\end{gathered}
$$

em que $q$ é o operador de atraso. A ordem relacionada com os polinômios $A(q), B(q)$ e $C(q)$ são de grande importância na definição do modelo que descreve o comportamento dinâmico desejado do sistema investigado. Deve ser lembrado, entretanto, que o modelo só capturará a dinâmica contida nos dados utilizados na modelagem condicionado ao poder de aproximação de sua representação (Aguirre, 2007).

Procurou-se, neste trabalho, obter modelos parcimoniosos e que descrevem a dinâmica contida nos dados tendo em vista somente o horizonte de predição. Detalhes de como os modelos foram obtidos podem ser obtidos dos autores. Optou-se por não divulgá-los neste trabalho uma vez que o foco está nas estratégias de predição.

\section{3 Índices de Desempenho}

Existem diversos índices de desempenho que são utilizados para quantificar a qualidade para predição de séries temporais (a qualidade do ajuste do modelo obtido a partir dos dados reais). Neste trabalho serão utilizados alguns índices listados em (Ribeiro, 2013), sendo que as definições reportadas logo abaixo são as mesmas descritas por ele em seu trabalho. 


\subsubsection{Erro Absoluto Médio (MAE)}

O MAE faz o cálculo do erro absoluto médio entre as séries simulada $(S)$ e observada $(O)$ a partir da equação 9:

$$
M A E=\frac{1}{N} \sum_{i=1}^{N}\left|\left(S_{i}-O_{i}\right)\right|
$$

\subsubsection{Erro Quadrático Médio (MSE)}

O MSE faz o cálculo do erro quadrático médio entre as séries simulada e observada por meio da seguinte equação 10 :

$$
M S E=\frac{1}{N} \sum_{i=1}^{N}\left(S_{i}-O_{i}\right)^{2}
$$

\subsubsection{Raiz Quadrada do Erro Quadrático Médio (RMSE)}

O RSME extrai a raiz quadrada do erro quadrático médio e pode ser representado conforme a equação 11 abaixo:

$$
R M S E=\sqrt{\frac{1}{N} \sum_{i=1}^{N}\left(S_{i}-O_{i}\right)^{2}}
$$

\subsubsection{Percent Bias (PBIAS)}

O índice PBIAS mede a tendência média da vazão simulada em relação à vazão observada, podendo ser maior ou menor que esta. $\mathrm{O}$ valor ideal para este índice é $0,0 \%$; um valor positivo indica uma tendência superestimada e um valor negativo indica uma tendência subestimada (Yapo, Gupta e Sorooshian, 1996). O valor retornado em percentual é dado pela equação 12 :

$$
P B I A S=100 \frac{\sum_{i=1}^{N}\left(S_{i}-O_{i}\right)}{\sum_{i=1}^{N} O_{i}}
$$

\subsubsection{RMSE - observations standard deviation (RSR)}

O RSR normaliza o índice RMSE utilizando o desvio padrão da série observada. Seu valor poderá variar de 0 a $\infty$, isso indica que as séries observada e simulada são iguais. Quanto menor o valor de RSR menor será o RMSE e melhor será o modelo ajustado. O índice RSR é calculado a partir da relação entre o RMSE e o desvio padrão da série observada, como apresentado na equação 13 :

$$
R S R=\frac{\sqrt{\sum_{i=1}^{N}\left(O_{i}-S_{i}\right)^{2}}}{\sqrt{\sum_{i=1}^{N}\left(O_{i}-\bar{O}\right)^{2}}}
$$

onde $\bar{O}$ representa a média de $O$.

\subsubsection{Eficiência de Nash-Sutcliffe (NSE)}

O RSE corresponde a uma estatística normalizada da variância do resíduo comparado com a variância dos dados observados. $N S E=1$ corresponde a uma aproximação perfeita dos dados observados, $1>N S E>0$ indica que a aproximação do modelo é melhor do que a média dos dados observados e $N S E<0$ indica que a média dos dados observados é um preditor melhor do que o modelo ajustado. A equação 14 que o representa é mostrada abaixo:

$$
N S E=1-\frac{\sum_{i=1}^{N}\left(s_{i}-o_{i}\right)^{2}}{\sum_{i=1}^{N}\left(O_{i}-\bar{O}\right)^{2}}
$$

\subsubsection{Erro Percentual Absoluto Médio (MAPE)}

O índice MAPE fornece uma medida da acurácia do modelo ajustado, seu retorno é dado em porcentagem $(\%)$ e quanto mais próximo de zero melhor é o modelo. Segue abaixo a equação 15 que o representa:

$$
M A P E=\frac{1}{N} \sum_{i=1}^{N}\left|\frac{O_{i}-S_{i}}{o_{i}}\right| x 100
$$

\section{Resultados e Discussões}

Nesta parte do trabalho os resultados são apresentados e discutidos de forma a se definir qual é a melhor estratégia utilizada no desenvolvimento do mesmo.

Utilizando a metodologia de modelagem brevemente descrita na seção anterior foram obtidos vários modelos dos quais apenas os melhores modelos foram usados na comparação mostrada no restante desta seção.

Os resultados foram dispostos em forma de tabela, na qual se compara os índices de desempenho para cada passo em um horizonte de até 10 passos à frente. As Tabelas 1, 2, 3, 4 e 5 mostram os índices de desempenho para as estratégias Direta, Recursiva, MIMO, DirRec e DIRMO respectivamente.

Tabela 1. - Apresentação dos Índices de Desempenho - Estratégia Direta.

\begin{tabular}{|c|c|c|c|c|c|c|c|}
\hline \multirow{2}{*}{ Passos (dias) } & \multicolumn{6}{|c|}{ Índice de desempenho da estratégia Direta } \\
\cline { 2 - 8 } & MAPE & MAE & MSE & RMSE & PBIAS & RSR & NSE \\
\hline 1 & 14,2481 & 6,7464 & 188,6061 & 13,7334 & 3,4066 & 0,3461 & 0,8802 \\
2 & 24,5074 & 10,8475 & 442,4382 & 21,0342 & 7,0060 & 0,5509 & 0,6965 \\
3 & 32,0927 & 13,3731 & 648,6842 & 25,4693 & 10,1937 & 0,6909 & 0,5226 \\
4 & 37,4026 & 14,7946 & 785,1579 & 28,0207 & 13,0162 & 0,7854 & 0,3832 \\
5 & 41,1032 & 15,6242 & 875,0065 & 29,5805 & 15,3292 & 0,8501 & 0,2773 \\
6 & 43,3998 & 16,0103 & 932,8598 & 30,5428 & 17,1587 & 0,8960 & 0,1972 \\
7 & 45,3437 & 16,4489 & 991,2680 & 31,4844 & 18,5196 & 0,9387 & 0,1189 \\
8 & 46,8062 & 16,7506 & 1035,8000 & 32,1844 & 19,7860 & 0,9733 & 0,0528 \\
9 & 48,4969 & 17,0635 & 1074,8000 & 32,7849 & 20,5601 & 0,9988 & 0,0023 \\
10 & 50,4129 & 17,4340 & 1110,8000 & 33,3286 & 21,3265 & 1,0214 & $-0,0433$ \\
\hline
\end{tabular}


Tabela 2. - Apresentação dos Índices de Desempenho - Estratégia Recursiva.

\begin{tabular}{|c|c|c|c|c|c|c|c|}
\hline \multirow[t]{2}{*}{ Passos (dias) } & \multicolumn{7}{|c|}{ Índice de desempenho da estratégia Recursiva } \\
\hline & MAPE & MAE & MSE & RMSE & PBIAS & RSR & NSE \\
\hline 1 & 14,2481 & 6,7464 & 188,6061 & 13,7334 & 3,4066 & 0,3461 & 0,8802 \\
\hline 2 & 19,3071 & 8,1719 & 217,6297 & 14,7523 & 6,7644 & 0,3794 & 0,8560 \\
\hline 3 & 25,2828 & 9,7132 & 262,0399 & 16,1876 & 9,5912 & 0,4166 & 0,8265 \\
\hline 4 & 32,0227 & 11,3682 & 324,1831 & 18,0051 & 12,3335 & 0,4605 & 0,7879 \\
\hline 5 & 39,5264 & 13,0783 & 402,7309 & 20,0682 & 15,0499 & 0,5081 & 0,7419 \\
\hline 6 & 47,6644 & 14,7666 & 491,9704 & 22,1804 & 17,6762 & 0,5538 & 0,6933 \\
\hline 7 & 56,4015 & 16,3791 & 588,2073 & 24,2530 & 20,2003 & 0,5956 & 0,6452 \\
\hline 8 & 65,7752 & 17,9029 & 689,0241 & 26,2493 & 22,6274 & 0,6332 & 0,5990 \\
\hline 9 & 75,8243 & 19,3419 & 792,3290 & 28,1483 & 24,9567 & 0,6666 & 0,5557 \\
\hline 10 & 86,5743 & 20,6821 & 896,3785 & 29,9396 & 27,1860 & 0,6959 & 0,5157 \\
\hline
\end{tabular}

Tabela 3. - Apresentação dos Índices de Desempenho - Estratégia MIMO

\begin{tabular}{|c|c|c|c|c|c|c|c|}
\hline \multirow{2}{*}{ Passos (dias) } & \multicolumn{5}{|c|}{ Índice de desempenho da estratégia MIMO } \\
\cline { 2 - 8 } & MAPE & MAE & MSE & RMSE & PBIAS & RSR & NSE \\
\hline 1 & 14,2415 & 6,7472 & 188,7764 & 13,7396 & 3,4092 & 0,3461 & 0,8802 \\
2 & 24,4993 & 10,8492 & 442,7974 & 21,0428 & 7,0049 & 0,5508 & 0,6966 \\
3 & 32,0837 & 13,3749 & 649,1470 & 25,4784 & 10,1891 & 0,6909 & 0,5227 \\
4 & 37,4000 & 14,7978 & 785,6745 & 28,0299 & 13,0084 & 0,7854 & 0,3832 \\
5 & 41,0931 & 15,6252 & 875,4549 & 29,5881 & 15,3220 & 0,8501 & 0,2774 \\
6 & 43,3775 & 16,0087 & 933,1457 & 30,5474 & 17,1506 & 0,8959 & 0,1973 \\
7 & 45,3294 & 16,4487 & 991,5155 & 31,4883 & 18,5078 & 0,9386 & 0,1189 \\
8 & 46,8054 & 16,7507 & 1036,1000 & 32,1879 & 19,7794 & 0,9732 & 0,0528 \\
9 & 48,5016 & 17,0648 & 1075,0000 & 32,7872 & 20,5605 & 0,9988 & 0,0023 \\
10 & 50,4129 & 17,4340 & 1110,8000 & 33,3286 & 21,3265 & 1,0214 & $-0,0433$ \\
\hline
\end{tabular}

Tabela 4. - Apresentação dos Índices de Desempenho - Estratégia DirRec.

\begin{tabular}{|c|c|c|c|c|c|c|c|}
\hline \multirow{2}{*}{ Passos (dias) } & \multicolumn{6}{|c|}{ Índice de desempenho da estratégia DirRec } \\
\cline { 2 - 8 } & MAPE & MAE & MSE & RMSE & PBIAS & RSR & NSE \\
\hline 1 & 14,2481 & 6,7464 & 188,6061 & 13,7334 & 3,4066 & 0,3461 & 0,8802 \\
2 & 21,2223 & 10,2995 & 463,5953 & 21,5313 & 3,7426 & 0,5371 & 0,7115 \\
3 & 26,9869 & 12,7744 & 704,4090 & 26,5407 & 3,5949 & 0,6622 & 0,5615 \\
4 & 31,0455 & 14,3896 & 869,7922 & 29,4922 & 3,3920 & 0,7372 & 0,4566 \\
5 & 33,5323 & 15,3760 & 974,8146 & 31,2220 & 3,2274 & 0,7793 & 0,3928 \\
6 & 35,3261 & 15,9453 & 1038,5000 & 32,2255 & 3,0669 & 0,8037 & 0,3541 \\
7 & 36,7402 & 16,4954 & 1106,7000 & 33,2669 & 2,8548 & 0,8282 & 0,3141 \\
8 & 38,1560 & 17,0117 & 1171,4000 & 34,2261 & 2,8163 & 0,8510 & 0,2757 \\
9 & 39,4625 & 17,5120 & 1236,8000 & 35,1687 & 2,6502 & 0,8727 & 0,2385 \\
10 & 40,8218 & 17,9581 & 1286,7000 & 35,8710 & 2,5817 & 0,8890 & 0,2097 \\
\hline
\end{tabular}

Tabela 5. - Apresentação dos Índices de Desempenho - Estratégia DIRMO.

\begin{tabular}{|c|c|c|c|c|c|c|c|}
\hline \multirow{2}{*}{ Passos (dias) } & \multicolumn{6}{|c|}{ Índice de desempenho da estratégia DIRMO } \\
\cline { 2 - 7 } & MAPE & MAE & MSE & RMSE & PBIAS & RSR & NSE \\
\hline 1 & 14,2415 & 6,7472 & 188,7764 & 13,7396 & 3,4092 & 0,3461 & 0,8802 \\
2 & 24,4993 & 10,8492 & 442,7974 & 21,0428 & 7,0049 & 0,5508 & 0,6966 \\
3 & 32,0837 & 13,3749 & 649,1470 & 25,4784 & 10,1891 & 0,6909 & 0,5227 \\
4 & 37,4000 & 14,7978 & 785,6745 & 28,0299 & 13,0084 & 0,7854 & 0,3832 \\
5 & 41,0931 & 15,6252 & 875,4549 & 29,5881 & 15,3220 & 0,8501 & 0,2774 \\
6 & 43,3775 & 16,0087 & 933,1457 & 30,5474 & 17,1506 & 0,8959 & 0,1973 \\
7 & 45,3294 & 16,4487 & 991,5155 & 31,4883 & 18,5078 & 0,9386 & 0,1189 \\
8 & 46,8054 & 16,7507 & 1036,1000 & 32,1879 & 19,7794 & 0,9732 & 0,0528 \\
9 & 48,5016 & 17,0648 & 1075,0000 & 32,7872 & 20,5605 & 0,9988 & 0,0023 \\
10 & 50,4129 & 17,4340 & 1110,8000 & 33,3286 & 21,3265 & 1,0214 & $-0,0433$ \\
\hline
\end{tabular}

De acordo com os resultados reportados nas tabelas pode-se ver que as estratégias Direta, MIMO e
DIRMO apresentaram o mesmo desempenho considerando todas as predições, ou seja, de 1 a 10 passos à frente. Por outro lado, quando as mesmas são comparadas às estratégias Recursiva e a DirRec, estas últimas apresentam desempenho superior.

Quando o índice MAPE é analisado, vê-se que de 1 até 10 passos, com exceção do segundo passo e do terceiro passo, a estratégia DirRec obteve valores menores, o que significa que uma mesma estratégia de modelagem pode gerar resultados diferentes e, portanto a estratégia de predição deve ser escolhida entre várias opções. No segundo e no terceiro passo a estratégia Recursiva teve um valor menor do que aqueles apresentados pela estratégia DirRec. Note, entretanto, que somente para esses passos a estratégia Recursiva obteve melhor resultado.

Ao se analisar o índice MAE, vê-se que de 1 até 7 passos a estratégia Recursiva teve menores valores de erro absoluto médio. E de 8 até 10 passos, a estratégia DirRec obteve valores menores.

Nos índices MSE e RME e RSR a estratégia Recursiva obteve valores menores se comparada com a DirRec. Isso significa que a estratégia Recursiva foi superior a DirRec nesses índices. Conjectura-se aqui que a estratégia Recursiva, por meio de seu modelo, pode melhor representar os dados em questão.

Ao se comparar os índices PBIAS e NSE, vê-se que a estratégia DirRec foi superior a Recursiva, o que reforça o comentário anterior.

As Figuras 4 e 5 mostram a previsão de 10 passos à frente para a Estratégia Recursiva e para a Estratégia DirRec. Note que na estratégia DirRec (Figura 5) a série observada foi melhor representada.

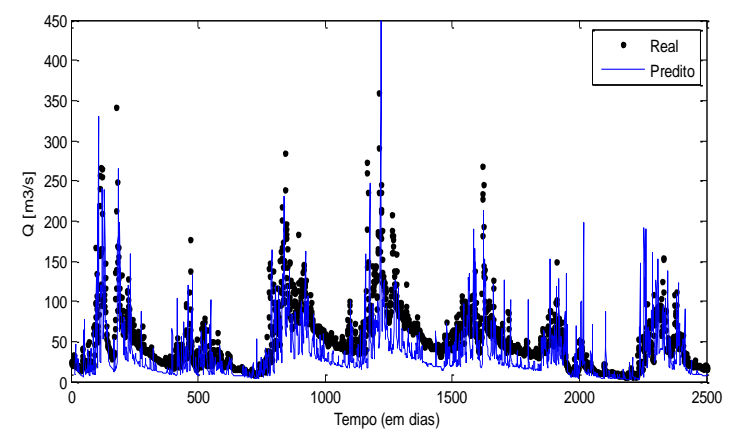

Figura 4: Predição de Vazão Q (dada em m3/s) - Estratégia Recursiva - 10 passos à frente

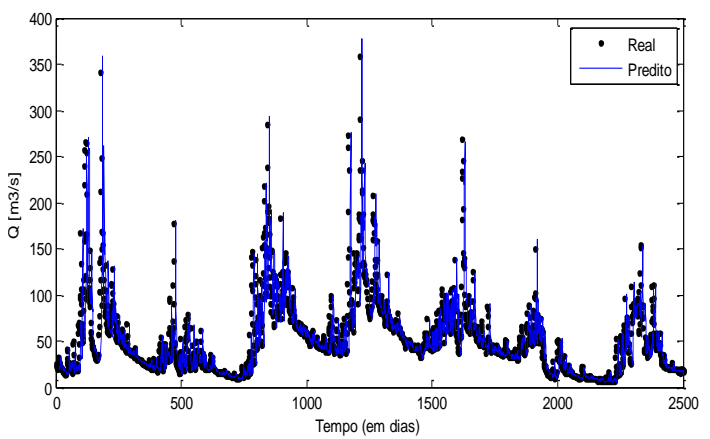

Figura 5: Predição de Vazão Q (dada em m3/s) - Estratégia DirRec - 10 passos à frente 


\section{Conclusão}

Predição de séries temporais para Multipassos à frente não é uma tarefa fácil, especialmente quando os dados coletados do sistema sob estudo não são bem amostrados. O objetivo deste trabalho foi analisar 5 tipos de estratégias e verificar qual delas obteve o melhor desempenho para predição de um horizonte de 10 passos à frente em uma bacia hidrográfica de pequeno porte, UHE Rosal no estado de Minas Gerais. Apesar do tamanho da amostra, os dados de vazão de afluência foram obtidos com amostragem diária, o que pode limitar o desempenho dos modelos obtidos.

Considerando uma metodologia específica para obtenção dos modelos, procurou-se explorar, neste trabalho, não o algoritmo de escolha de modelos ou representação em si, mas a estratégia para predição de vários passos à frente.

Do ponto específico de aplicação, mostrou-se na seção 4 que a estratégia Recursiva se torna interessante quando necessita-se de um menor horizonte de predição de vazão afluente. Entretanto o mesmo não se repete quando o horizonte é maior.

Tendo em vista que, para as concessionárias de energia, o índice de desempenho MAPE é o mais interessante, pode-se dizer que a estratégia DirRec é a melhor neste caso específico.

Finalmente os resultados apresentados neste trabalho indicam que vale a pena considerar diferentes estratégias para predição de vários passos à frente. Isso aliado a escolha de representação de sistemas e de algoritmos de estimação de parâmetros pode levar a obtenção de modelos com melhores índices de desempenho.

\section{Agradecimentos}

Os autores agradecem o apoio da CEMIG GT, FAPEMIG, CAPES e CNPq.

\section{Referências Bibliográficas}

Aguirre, L. A. (2007). Introdução à Identificação de Sistemas. 3rd, Editora UFMG, Belo Horizonte, (p.130).

Ben Taieb, S., Bontempi, G., Sorjamaa, A., \& Lendasse, A. (2009). Long-term prediction of time series by combining direct and mimo strategies. In International joint conference on neural networks.

$<$ http://eprints.pascalnetwork.org/archive/00004 925/>.

Yapo P. O, Gupta H. V., and Soroosh Sorooshian. Automatic calibration of conceptual rainfallruno_ models: sensitivity to calibration data. Journal of Hydrology, 181(1-4):23 - 48, 1996. DOI: 10.1016/0022-1694(95)02918-4

Bontempi, G. (2008). Long term time series prediction with multi-input multi-output local learning. In Proceedings of the 2nd European symposium on time series prediction (TSP), ESTSP08, Helsinki, Finland (pp. 145-154).

DOI: 10.1016/j.ijforecast.2010.09.004

Ribeiro F. M., Sistema de Previsão de Afluência Utilizando Árvores de Regressão Linear Nebulosas Evolutivas. 2013.88f. Monografia (Graduação)- Universidade Federal de Minas Gerais UFMG, Belo Horizonte, 2013

Bontempi, G., \& Ben Taieb, S. (2011). Conditionally dependent strategies for multiple-step-ahead prediction in local learning. International Journal of Forecasting, 27(3), 689-699.

Ben Taieb, S. et all (2012), A review and comparison of strategies for multi-step ahead time series forecasting based on the NN5 forecasting competition. Expert Systems with Aplications, pp. 767-7083.

Sorjamaa, A., \& Lendasse, A. (2006). Time series prediction using dirrec strategy. In M. Verleysen (Ed.), ESANN06, European symposium on artificial neural networks, Bruges, Belgium (pp. 143-148).

Sorjamaa, A., Hao, J., Reyhani, N., Ji, Y., \& Lendasse, A. (2007). Methodology for longterm prediction of time series. Neurocomputing, 70(16-18), 2861-2869. DOI: 10.1016/j.neucom.2006.06.015

Chevillon G. (2007). Direct Multi-step Estimation and Forecasting. Journal of Economic Surveys. Volume 21, Issue 4, Publisher Wiley Online Library (p. 746-785). DOI: 10.1111/j.1467-6419.2007.00518.x

Marcellino M., Stock J., Watson M. (2006), A comparison of Direct and Iterated Multistep AR methods for Forecasting Macroeconomic Time Series. Journaal of Econometrics, volume 135, Issue 1-2, Publisher Elsevier (p. 499-526).

Parag P., bhagwat, Maity R. (2012), Multi-Ahead River Flow Prediction Using LS-SVR at Daily Scale. Journal of Water Resource and $\mathrm{P}$, rotection, 4, p. 528-539. DOI: 10.4236/jwarp.2012.47062

Pianosi F., and Raso L., Dynamic modeling of predictive uncertainty by regression on absolute errors. Water Resources Research, VOL. 48, W03516, doi:10.1029/2011WR010603, 2012. DOI: 10.1029/2011WR010603

Bao Y., Member, IEEE, Xiong T., Hu Z (2012). Mutiple-Ooutput Support Vector Regression. IEE Transactions on Neural Networks and Learning Systems.

Box G. E. P., Jenkins G. M., Reinsel G. C. Time Series Analysis: Forecasting and Control. Wiley, jun $30,2008,784$ p. 